\title{
Study of the Static and Dynamic Nuclear Properties and Form Factors for Some Magnesium Isotopes ${ }^{29-34} \mathrm{Mg}$
}

\section{Lubna Abduljabbar Mahmood, Ghaith Naima Flaiyh}

Department of Physics, College of Science, Baghdad University, Baghdad, Iraq

E-mail: ghaithnaima79@gmail.com

Corresponding author: lubna917@yahoo.com

\begin{abstract}
Nuclear structure of ${ }^{29-34} \mathrm{Mg}$ isotopes have been investigated using shell model with Skyrme-Hartree-Fock calculations. In particular, nuclear density distributions for proton, neutron, mass and charge densities with their corresponding root mean square radii, neutron skin thicknesses and inelastic electron scattering form factors were calculated for positive low-lying states. The deduced results were discussed for the longitudinal and transverse form factors and compared with the available experimental data. It was confirmed that the combining shell model with Hartree-Fock mean field method with Skyrme interaction can accommodate very well with the nuclear excitation properties of stable and unstable nuclei.
\end{abstract}

Key words

Shell model, charge density, longitudinal form factors, transverses form factors.

\section{Article info.}

Received: Jan. 2021

Accepted: Mar. 2021

Published: Jun. 2021

\section{دراسة الخواص النووية المستقرة والديناميكية وعوامل الشكل لبعض نظائر المغنيسيوم 29-34 Mg

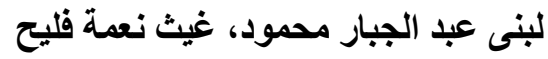$$
\text { قسم الفيزياء، كلية العلوم، جامعة بغداد، بغداد، العر اق }
$$

\footnotetext{
Skyrme- تم دراسة النركيب النووي للنظائر و Hartree-Fock

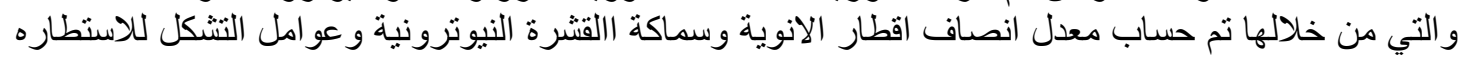

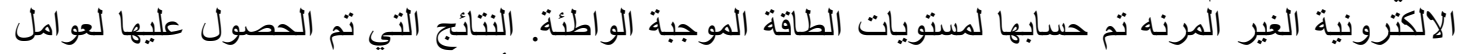

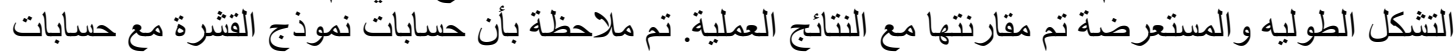
Skyrme-Hartree
}

\section{Introduction}

In atomic shell model, the shells are characterized according to atomic quantum numbers which may be computed from atomic Coulomb potential (and ensuing the equation of the Eigen value) as given by the protons of the nucleus.

The shells are filled with nucleons so as to increase the energy values, in a way that every orbit (i.e. levels) may contain at the most two nucleons (according to Pauli Exclusion Principle). The atom characteristics are then mainly specified by the 
nucleons in non-entirely filled shells. Which results in atomic characteristics periodicity, like atomic radius and energy of the ionization, which is reflected in the elements' periodic table [1].

The nuclear shell model is partially similar to the atomic shell model, describing the electrons' arrangement in the atom, which means that a filled shell produces higher stability. In the atomic shell model [2]. Specific landmarks, "magic number", which are related to the "inert" nuclei, dominated nuclear landscapes for more than five decades.

Several studies have been performed to investigate the nuclear structure of magnesium isotopes [3-5] such as, nuclear densities for proton, neutron, mass and charge densities with their corresponding rms radii, neutron skin thicknesses and inelastic electron scattering form factors.

The present work is dedicated to study the ground properties (rms, neutron skin thicknesses and nuclear density distributions) and inelastic electron scattering form factors (Longitudinal and Transverse) for some Magnesium isotopes ${ }^{29-34} \mathrm{Mg}$.

\section{Theory}

In the nuclear density distribution (NDD), it is considered that nucleus nucleons play the role of point particles. The nucleus nucleon density, which consists of A point-like particles has been characterized based on operators [6]

$$
\rho_{0}^{(1)}(r)=2 \sum_{n \ell} \frac{2(2 \ell+1)}{4 \pi}\left|R_{n \ell}(r)\right|^{2}
$$

where $R_{n l}(r)$ is the radial part of the wave function, the quantity inside the summation of Eq. (1) represents the charge density distributions (CDD) of a nucleus. Thus the ground state CDD's of one body operator for closed shell nuclei with $Z=N$ can be deduced from the ground state NDD of Eq. (1), i.e. CDD=1/2 NDD, and is given by

$$
\rho_{c h}^{(1)}(r)=\sum_{n \ell} \frac{2(2 \ell+1)}{4 \pi}\left|R_{n \ell}(r)\right|^{2}
$$

in electronic charge per volume units $\left(\mathrm{e} / \mathrm{fm}^{3}\right)$.

It should be remarked that the occupation number of protons $2(2 \ell+1)$ of the orbit $\ell$ can be written in terms of the occupation number of sub-orbits $(2 j+1)$, i.e.

$$
2(2 \ell+1)=\sum_{j}(2 j+1)
$$

Introducing Eq. (2) into (3), we get

$$
\rho_{c h}^{(1)}(r)=\frac{1}{4 \pi} \sum_{n \ell j}(2 j+1)\left|R_{n \ell}(r)\right|^{2}
$$

It is possible to rewrite Eq. (4) as

$$
\rho_{c h}^{(1)}(r)=\frac{1}{4 \pi} \sum_{n \ell j} \eta_{n \ell j}(2 j+1)\left|R_{n \ell}(r)\right|^{2}
$$

where a parameter $\eta_{n \ell j}$ can be defined as a state $n \ell j$ occupation probability. $\eta_{n \ell j}=0$ or 1 for the closed shell nuclei with $Z=N$ whereas for the open shell nuclei (with $Z=N$ ) we use the same expression of Eq. (5) but now $0<\eta_{n \ell j}<1$. In this way the calculations are extended to include the open shell nuclei in our study [6].

In a PWBA (i.e. plane-wave Born approximation), differential cross-section for electron scattering from nucleus that has charge $(\mathrm{Ze})$ and mass $(\mathrm{M})$ into a solid angle $(\mathrm{d} \Omega)$ is given by [7]: 


$$
\frac{d \sigma}{d \Omega}=\left(\frac{d \sigma}{d \Omega}\right)_{M o t t} f_{r e c} \sum_{J}\left|F_{J}(q, \theta)\right|^{2}
$$

$\left(\frac{d \sigma}{d \Omega}\right)_{M o t t}$ is the Mott's cross-section, which is the scattering of a relativistic electron with high energies from a spinless point charge, and is given by [8]:

$$
\left(\frac{d \sigma}{d \Omega}\right)_{M o t t}=\left(\frac{z e^{2} \cos \left(\frac{\theta}{2}\right)}{2 E_{i} \hbar c \sin ^{2}\left(\frac{\theta}{2}\right)}\right)^{2}
$$

and the nuclear recoil factor is given by [8]

$$
f_{r e c}=\left(1+\frac{2 E_{i}}{M} \sin ^{2}\left(\frac{\theta}{2}\right)\right)^{-1}
$$

The total form factor $F_{J}(q, \theta)$ of a given multipolarity $J$ consists of a Longitudinal (Coulomb) part $\left(F_{J}^{L}\right)$ and a Transverse part $\left(F_{J}^{T}\right)$ defined as [9]:

$$
F_{J}^{2}(q, \theta)=\left(\frac{q_{\mu}}{q}\right)^{4}\left|F_{J}^{L}(q)\right|^{2}+\left[\frac{q_{\mu}^{2}}{2 q^{2}}+\tan ^{2}\left(\frac{\theta}{2}\right)\right]\left|F_{J}^{T}(q)\right|^{2}
$$

And

$$
\left|F_{J}^{T}(q)\right|^{2}=\left|F_{J}^{E l}(q)\right|^{2}+\left|F_{J}^{m a g}(q)\right|^{2}
$$

The form factor of multipolarity $(J)$ as a function of momentum transfer is written in terms of the reduced matrix elements of the transition operator as [8]:

$$
\left|F_{J}^{\eta}(q)\right|^{2}=\frac{4 \pi}{z^{2}\left(2 J_{i}+1\right)}\left|\left\langle J_{f}|| T_{J}^{\eta}(q)|| J_{i}\right\rangle\right|^{2}
$$

The nuclear states have a well-defined isospin. Therefore, utilizing Wigner-Eckart theorem in the isospin space, the form factor can be expressed as a matrix element which is reduced in the total angular momentum (spin) (J) as well as isospin (T) (i.e. the elements of the triple-bar matrix). In addition to that, in realistic form factor calculations, there is a necessity to be taken under consideration which are the impacts of the finite size (f.s), Coulomb distortion, and center of mass (c.m) motion of the electron waves. Which is why, form factor for a certain multi-polarity $(\mathbf{J})$ may be expressed based on matrix elements that are reduced in the spin space as well as the iso-spin space as:

$$
\begin{aligned}
\left|F_{J}^{\eta}(q)\right|^{2}= & \frac{4 \pi}{Z^{2}\left(2 J_{i}+1\right)}\left|\sum_{T=0,1}(-1)^{T_{f}-T_{Z f}}\left(\begin{array}{ccc}
T_{f} & T & T_{i} \\
-T_{Z f} & M_{T} & T_{Z i}
\end{array}\right)\left\langle\Gamma_{f}\left|\left\|T_{J, T}^{\mu}(q)\right\|\right| \Gamma_{i}\right\rangle\right|^{2} \\
& \times\left|F_{c m}(q)\right|^{2} \times\left|F_{f . s}(q)\right|^{2}
\end{aligned}
$$

The multipolarity $(\mathrm{J})$ in the last equation is restricted by angular momentum selection rule [9]:

$$
\left|J_{i}-J_{f}\right| \leq J \leq J_{i}+J_{f} \quad \triangle\left(J_{i}, J_{f}, J\right)
$$

And 


$$
\left|T_{i}-T_{f}\right| \leq T \leq T_{i}+T_{f}
$$

The parity selection rules (for same parity)

$$
\begin{aligned}
& \triangle \pi^{E l}=(-1)^{J} \\
& \triangle \pi^{m a g}=(-1)^{J+1}
\end{aligned}
$$

\section{Results and discussion}

Fig. 1 illustrates the relation between the number of nucleons and the root mean square for the charge of ${ }^{29-34} \mathrm{Mg}$ nuclei. It is noted that the root mean square of the charge increases when the number of nucleons increases (number of neutron increases). The results are given in Table 1.

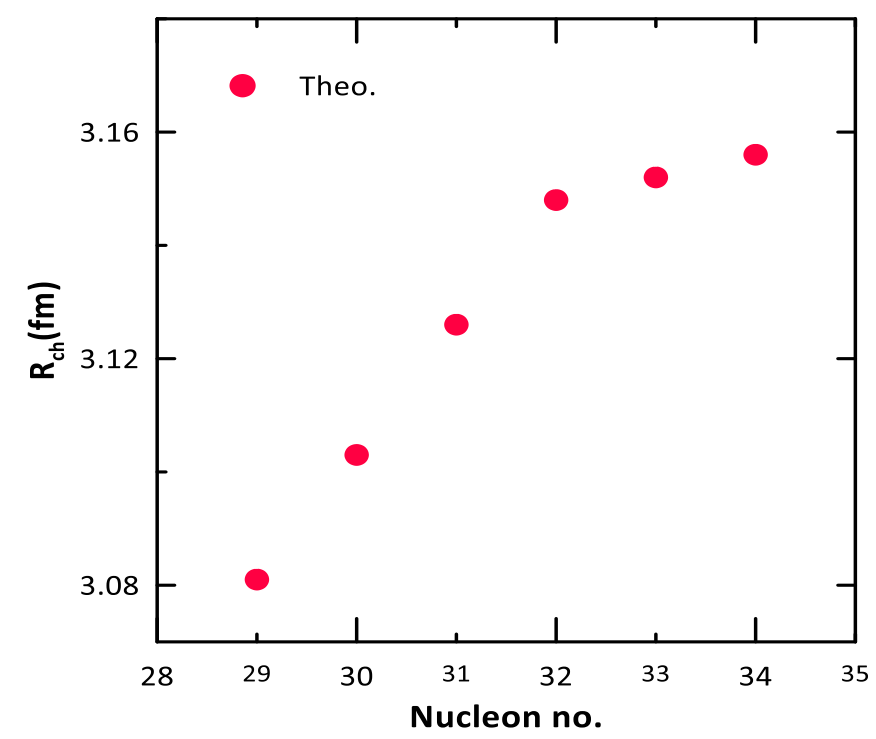

Fig. 1: The relation between the rms for charge and the number of nucleon of ${ }^{29-34} \mathrm{Mg}$ nuclei.

Table 1: The values of the root mean square for charge of ${ }^{29-34} \mathrm{Mg}$ nuclei.

\begin{tabular}{|c|c|}
\hline Nuclei & Rch (fm)Theory \\
\hline${ }^{29} \mathbf{M g}$ & 3.081 \\
\hline${ }^{30} \mathbf{M g}$ & 3.103 \\
\hline${ }^{31} \mathbf{M g}$ & 3.126 \\
\hline${ }^{32} \mathbf{M g}$ & 3.148 \\
\hline${ }^{33} \mathbf{M g}$ & 3.152 \\
\hline${ }^{34} \mathbf{M g}$ & 3.156 \\
\hline
\end{tabular}

Fig. 2 shows the relation between the number of nucleons and the neutron skin thickness of ${ }^{29-34} \mathrm{Mg}$ nuclei. It is noted the neutron skin thickness increases when the number of nucleons increases. The results are given in Table 2. 


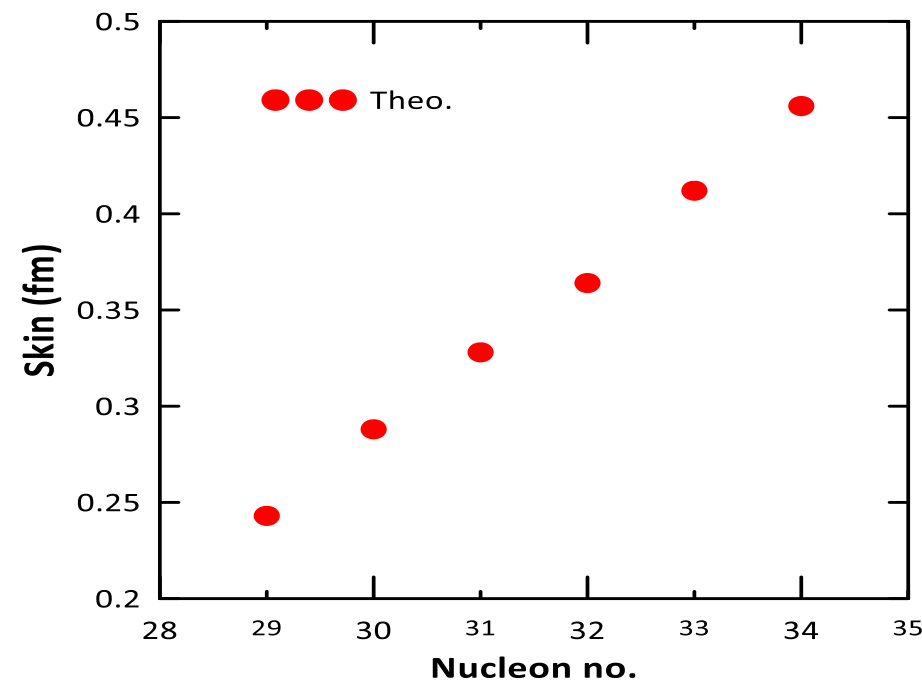

Fig. 2: Relation between the number of nucleons and the neutron skin thickness for ${ }^{29-34} \mathrm{Mg}$ nuclei.

Table 2: The values of the neutron skin thickness for ${ }^{29-34} \mathrm{Mg}$ nuclei.

\begin{tabular}{|c|c|}
\hline Nuclei & Skin(fm) \\
\hline${ }^{29} \mathbf{M g}$ & 0.243 \\
\hline${ }^{30} \mathbf{M g}$ & 0.288 \\
\hline${ }^{31} \mathbf{M g}$ & 0.328 \\
\hline${ }^{32} \mathbf{M g}$ & 0.364 \\
\hline${ }^{33} \mathbf{M g}$ & 0.412 \\
\hline${ }^{34} \mathbf{M g}$ & 0.456 \\
\hline
\end{tabular}

Fig. 3 shows the relation between the number of nucleons and radius for protons, neutrons and matter. It can be seen that as the number of nucleons increases, the radii of protons, neutrons and matter increases. The radii of neutrons are larger than that of protons and matter because the mass of the neutron is more than the mass of the proton. The results are given in Table 3.

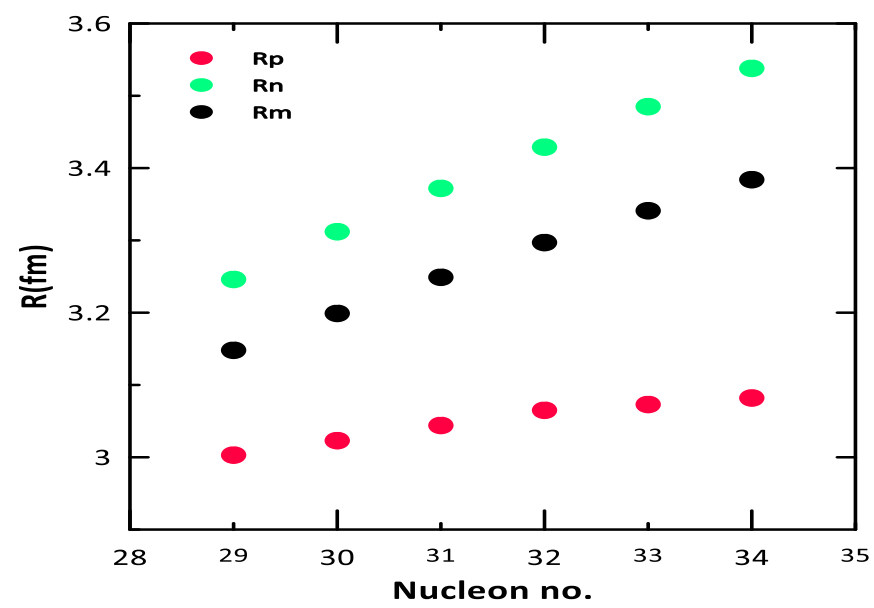

Fig. 3: The relation between radius and number of nucleon of ${ }^{29-34} \mathrm{Mg}$ nuclei. 
Table 3: The values of radii of protons, neutrons and matter of ${ }^{29-34} \mathrm{Mg}$ nuclei.

\begin{tabular}{|c|c|c|c|}
\hline Nuclei & $\mathbf{R p}(\mathbf{f m})$ & $\mathbf{R n}(\mathbf{f m})$ & $\mathbf{R m}(\mathbf{f m})$ \\
\hline${ }^{29} \mathbf{M g}$ & 3.003 & 3.246 & 3.148 \\
\hline${ }^{30} \mathbf{M g}$ & 3.023 & 3.312 & 3.199 \\
\hline${ }^{31} \mathbf{M g}$ & 3.044 & 3.372 & 3.249 \\
\hline${ }^{32} \mathbf{M g}$ & 3.065 & 3.429 & 3.297 \\
\hline${ }^{33} \mathbf{M g}$ & 3.073 & 3.485 & 3.341 \\
\hline${ }^{34} \mathbf{M g}$ & 3.082 & 3.538 & 3.384 \\
\hline
\end{tabular}

Fig.4(a) shows the relation between charge density distributions and position $\mathrm{r}(\mathrm{fm})$ for $\mathrm{Mg}^{29,31,33}$ nuclei. It is noted that charge density distributions for ${ }^{29} \mathrm{Mg}$ are higher than those for ${ }^{31,33} \mathrm{Mg}$ in the center region about $(0.074 \mathrm{fm})$. the increase in the number of neutrons leads to decrease in the density of the charge in the center region where the charge density for ${ }^{29} \mathrm{Mg}$ higher than from ${ }^{31,33} \mathrm{Mg}$, due to the increase in the number of neutrons in the nucleus ${ }^{31,33} \mathrm{Mg}$, which leads to this deficiency.

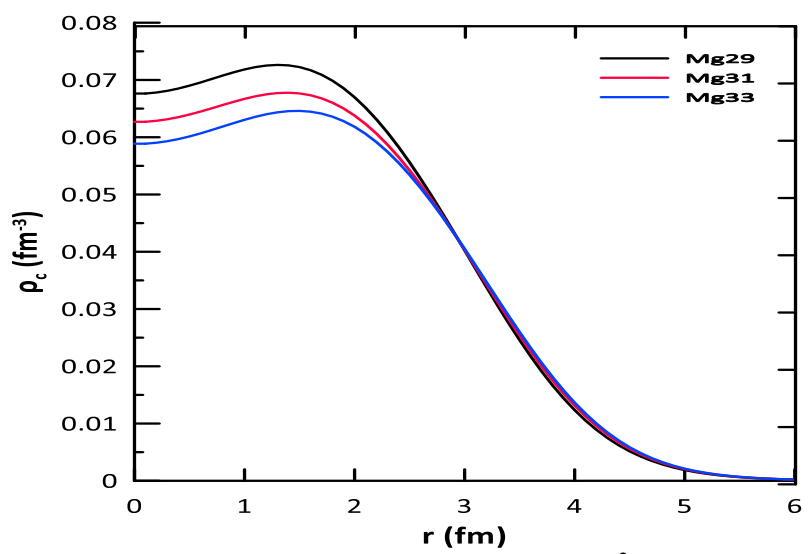

Fig. 4(a): The relation between charge density $\left(\mathrm{fm}^{-3}\right)$ and position $r(f m)$ for ${ }^{29,31,33} \mathrm{Mg}$ nuclei.

Fig. 4(b) shows the relation between charge density distributions and position $\mathrm{r}(\mathrm{fm})$ for ${ }^{30,32,34} \mathrm{Mg}$ nuclei. It can be noted that charge density distributions for ${ }^{30} \mathrm{Mg}$ are the highest in the center region $(0.07 \mathrm{fm})$. It is also noted that the increase in the number of neutrons leads to a decrease in the charge density in the center region, where the charge density distributions for ${ }^{30} \mathrm{Mg}$ are higher than that for ${ }^{34} \mathrm{Mg}$, due to the increase in the number of neutrons in the nucleus ${ }^{34} \mathrm{Mg}$, which leads to this deficiency.

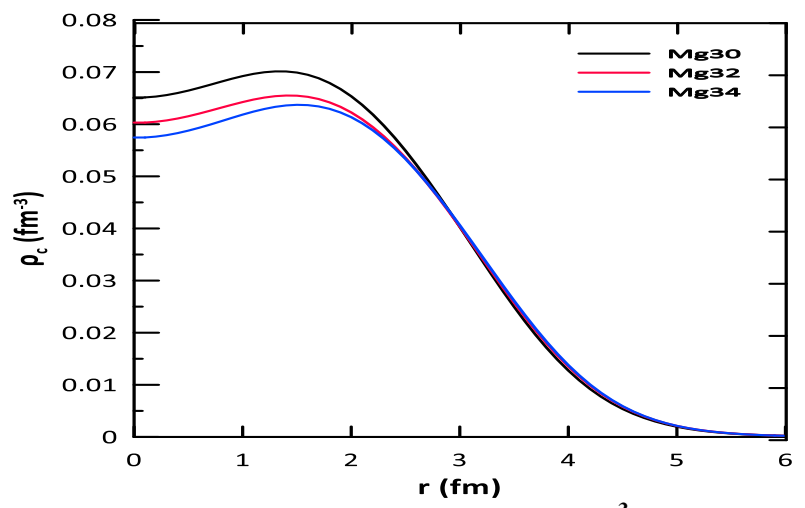
Fig. 4(b): The relation between charge density $\left(\mathrm{fm}^{-3}\right)$ and position $\mathrm{r}(\mathrm{fm})$ for

Fig. 5(a) shows the relation between proton density distributions and position $\mathrm{r}(\mathrm{fm})$ for ${ }^{29,31,33} \mathrm{Mg}$ nuclei. The proton density distributions for ${ }^{29} \mathrm{Mg}$ are the highest in the 
center region $(0.077 \mathrm{fm})$. It can be noted that the increase in the number of neutrons leads to a decrease in the proton density in the center region. The proton density for ${ }^{29} \mathrm{Mg}$ is higher than that for ${ }^{33} \mathrm{Mg}$, due to the increase in the number of neutrons in the nucleus ${ }^{33} \mathrm{Mg}$, which leads to this deficiency.

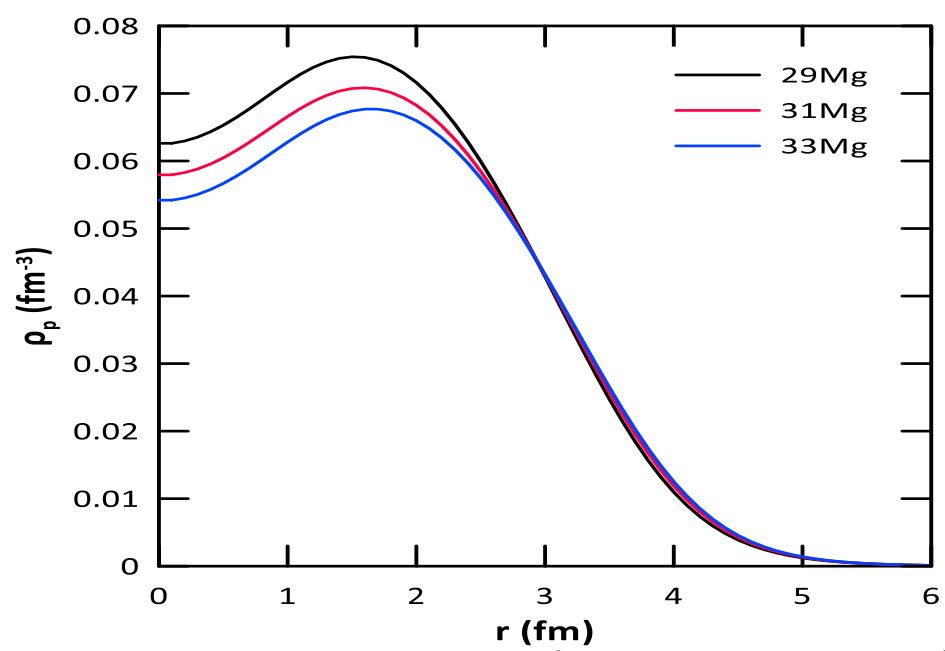

Fig. 5(a): The relation between proton density $\left(\mathrm{fm}^{-3}\right)$ and position $r(\mathrm{fm})$ for ${ }^{29,31,33} \mathrm{Mg}$ nuclei.

Fig. 5(b) shows the relation between proton density and position $\mathrm{r}(\mathrm{fm})$ for ${ }^{30,32,34} \mathrm{Mg}$. Proton density distributions for ${ }^{30} \mathrm{Mg}$ are the highest in the center region ( $\mathrm{r}$ $<2 \mathrm{fm}$ ) while the lowest value is for ${ }^{34} \mathrm{Mg}$ (about $0.053 \mathrm{fm}$ ). The increase in the number of neutrons leads to a decrease in the proton density in the region $r<2 \mathrm{fm}$ where the proton density for ${ }^{30} \mathrm{Mg}$ is higher than that for ${ }^{34} \mathrm{Mg}$, due to the increase in the number of neutrons in the ${ }^{34} \mathrm{Mg}$ nucleus, which leads to this deficiency.

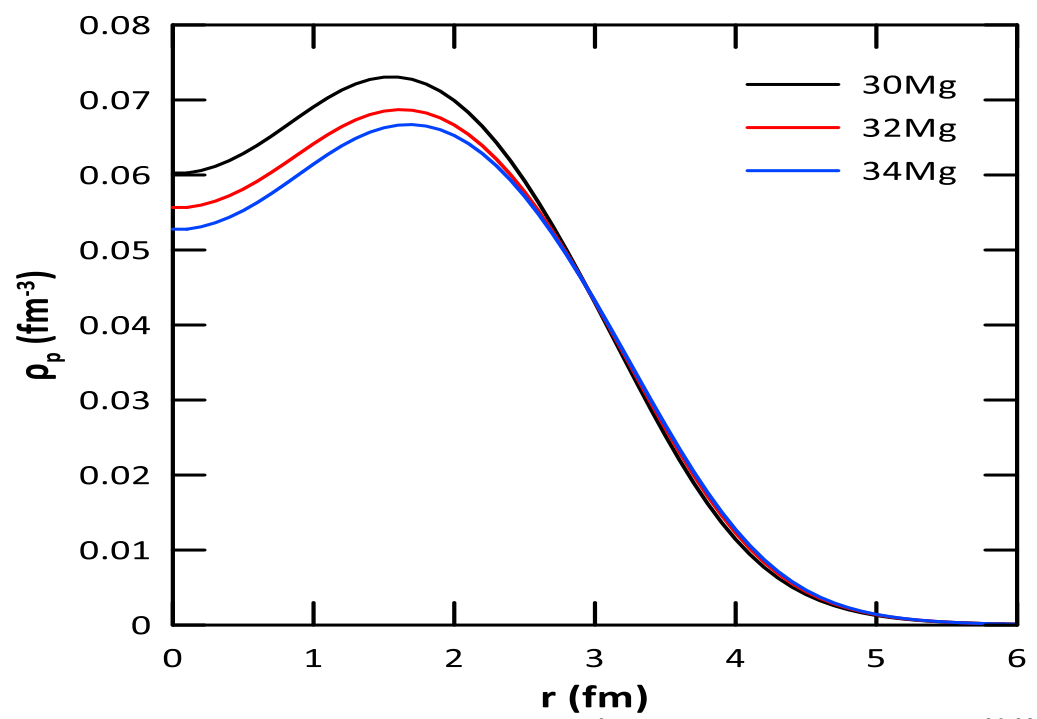

Fig. 5(b): The relation between proton density $\left(\mathrm{fm}^{-3}\right)$ and position $r(f m)$ for ${ }^{30,32,34} \mathrm{Mg}$ nuclei.

Fig. 6(a) shows the relation between neutron density distributions and the position $\mathrm{r}(\mathrm{fm})$ for ${ }^{29,31,33} \mathrm{Mg}$ nuclei. Neutron density for ${ }^{29} \mathrm{Mg}$ is the highest in the center region $\mathrm{r}<2 \mathrm{fm}$. 


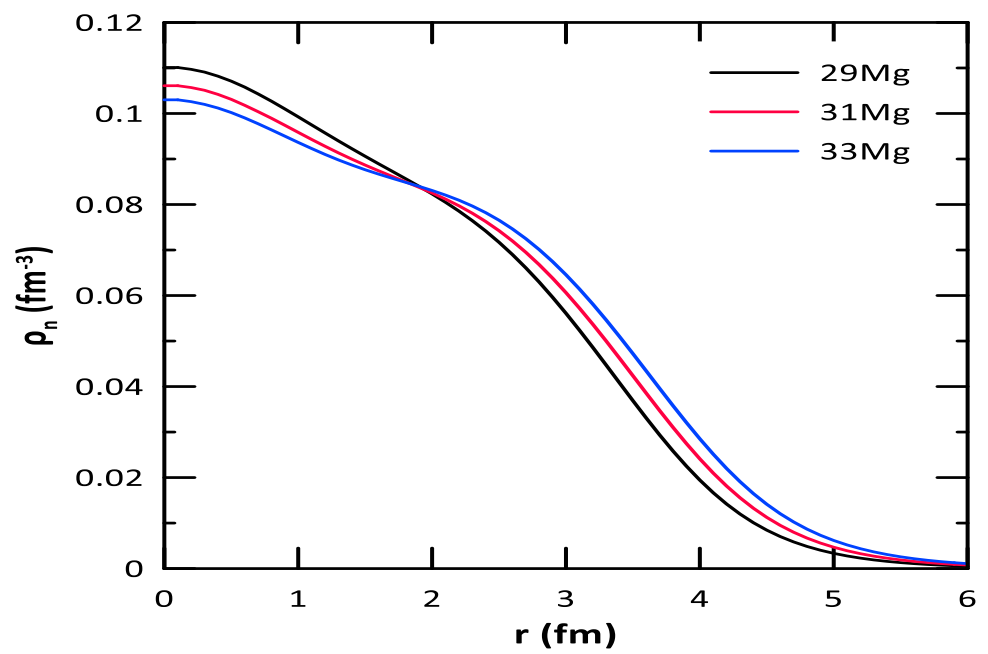

Fig. 6(a): The relation between neutron density $\left(\mathrm{fm}^{-3}\right)$ and position $r(f m)$ for ${ }^{29,31,33} \mathrm{Mg}$ nuclei.

Fig.6(b) shows the relation between neutron density and position $r(f m)$, for ${ }^{30,32,34} \mathrm{Mg}$ nuclei. Neutron density for ${ }^{30} \mathrm{Mg}$ is the highest in the center region $\mathrm{r}<2 \mathrm{fm}$.

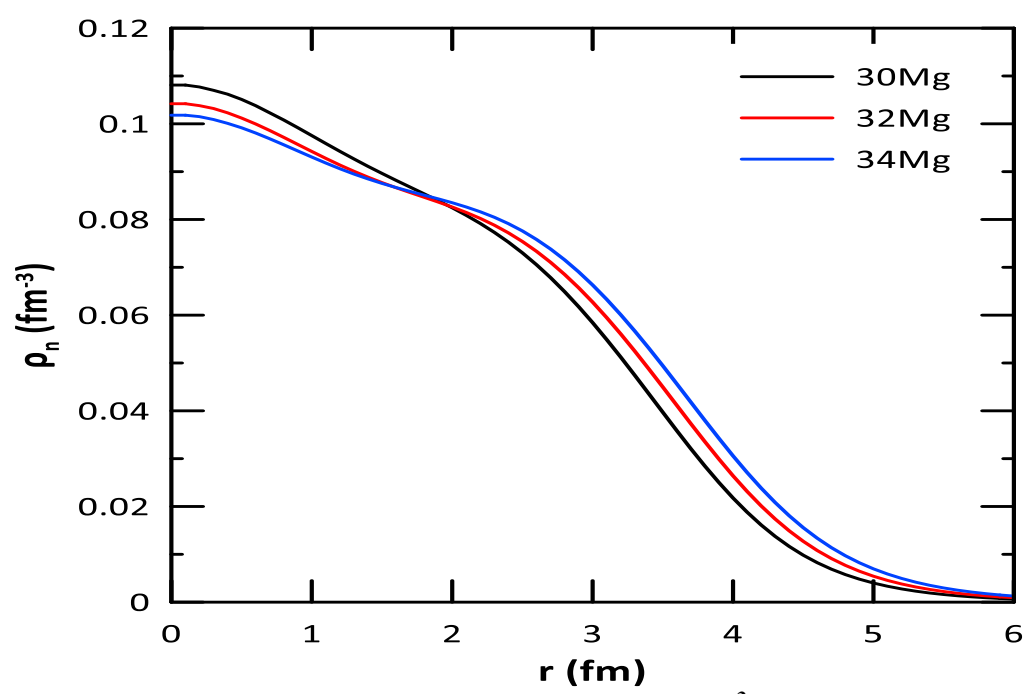

Fig. 6(b): The relation between neutron density $\left(\mathrm{fm}^{-3}\right)$ and position $r(f m)$ for ${ }^{30,32,34} \mathrm{Mg}$ nuclei.

Fig.7(a) shows the relation between matter density for $\mathrm{Mg}^{29,31,33}$ nuclei and position $\mathrm{r}(\mathrm{fm})$. It is noted from this figure that matter density for ${ }^{29} \mathrm{Mg}$ is the highest in the region $\mathrm{r}<2 \mathrm{fm}$ and the lowest for ${ }^{33} \mathrm{Mg}$. The increase in the number of neutrons leads to a decrease in the matter density in the center region where the matter density for ${ }^{29} \mathrm{Mg}$ is higher than that for ${ }^{33} \mathrm{Mg}$, due to the increase in the number of neutrons in the ${ }^{33} \mathrm{Mg}$ nucleus, which leads to this deficiency. 


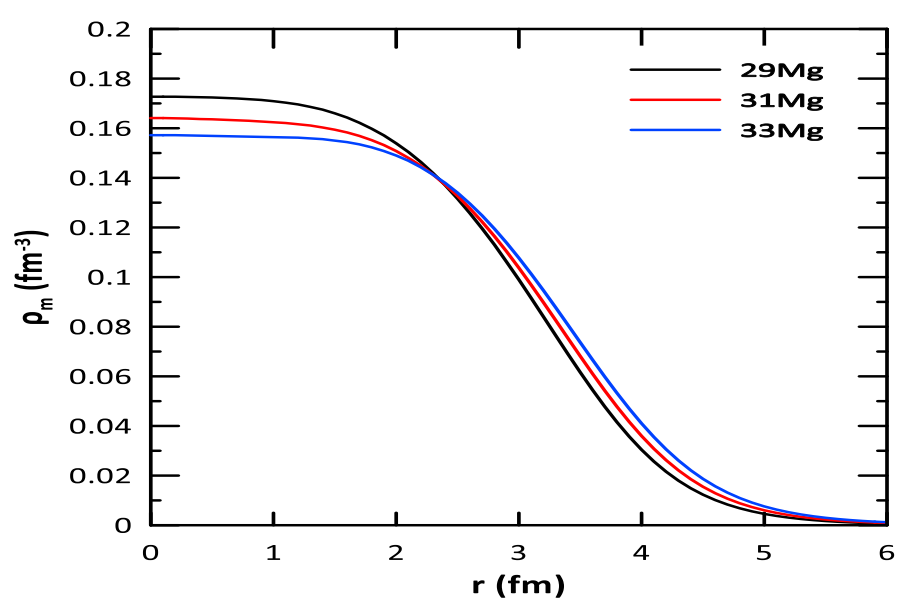

Fig. 7(a): The relation between matter density $\left(\mathrm{fm}^{-3}\right)$ and position $r(f m)$ for ${ }^{29,31,33} \mathrm{Mg}$ nuclei.

Fig. 7(b) shows the relation between matter density and position $r(f m)$ for ${ }^{30,32,34} \mathrm{Mg}$ nuclei. From this figure, it can be seen that in the center region $\mathrm{r}<2 \mathrm{fm}$, ${ }^{30} \mathrm{Mg}$ has the highest matter density while the lowest is for ${ }^{34} \mathrm{Mg}$. The increase in the number of neutrons leads to a decrease in the matter density in the center region. The matter density for ${ }^{30} \mathrm{Mg}$ being higher than that for ${ }^{34} \mathrm{Mg}$, due to the increase in the number of neutrons in the nucleus ${ }^{34} \mathrm{Mg}$, which leads to this deficiency.

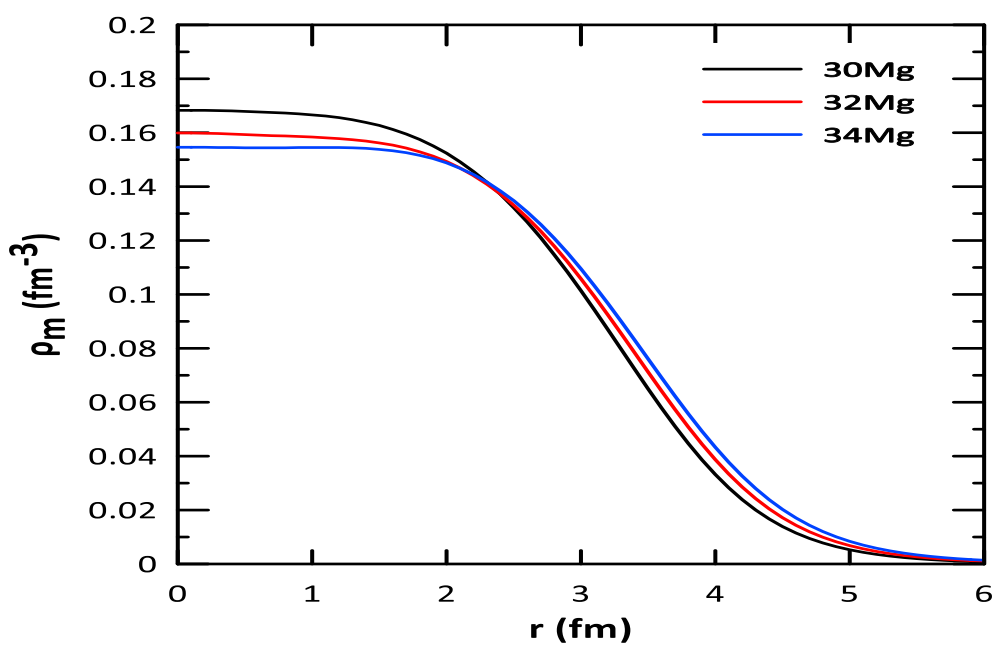

Fig. 7(b): The relation between matter density $\left(\mathrm{fm}^{-3}\right)$ and position $r(\mathrm{fm})$ for ${ }^{30,32,34} \mathrm{Mg}$ nuclei.

Fig. 8 shows the relation between moment transfer and longitudinal form factor for ${ }^{25,29,31} \mathrm{Mg}$ nuclei. The longitudinal form factor of ${ }^{25} \mathrm{Mg}$ nuclei for the transition $\left(J_{i}^{\pi} T_{i}=5 / 2^{+} 1 / 2\right)$ to the $\left(J_{f}^{\pi} T_{f}=7 / 2^{+} 1 / 2\right)$ at $1.739 \mathrm{MeV}$ is represented by red solid line. The longitudinal form factor of ${ }^{29} \mathrm{Mg}$ nuclei for the transition $\left(J_{i}^{\pi} T_{i}=\right.$ $\left.3 / 2^{+} 1 / 2\right)$ to the $\left(J_{f}^{\pi} T_{f}=1 / 2^{+} 1 / 2\right)$ at $0.088 \mathrm{MeV}$ is represented by black solid line. As for the magnesium isotope ${ }^{31} \mathrm{Mg}$ the transition $\left(J_{i}^{\pi} T_{i}=1 / 2^{+} 1 / 2\right)$ to the $\left(J_{f}^{\pi} T_{f}=3 / 2^{+} 1 / 2\right)$ at $0.176 \mathrm{MeV}$ is represented by black dashed line. 


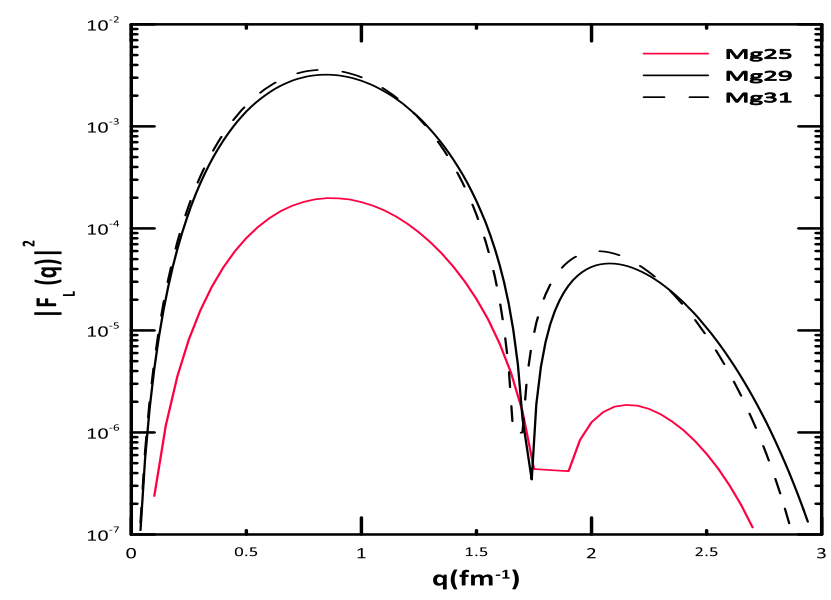

Fig. 8: The relation between moment transfer and longitudinal form factor for ${ }^{25,29,31} \mathrm{Mg}$ nuclei.

Fig. 9 shows the relation between moment transfer and longitudinal form factor. In this figure, the theoretical results of the isotopes of magnesium ${ }^{24,30,32,34} \mathrm{Mg}$ are compared with $\mathrm{Li}$ et al. [10] experimental results at $1.369 \mathrm{MeV}$ for the magnesium isotope ${ }^{24} \mathrm{Mg}$ (represented by the red circles). The transition for ${ }^{24} \mathrm{Mg}\left(J_{i}^{\pi} T_{i}=0^{+} 0\right)$ to the $\left(J_{f}^{\pi} T_{f}=2^{+} 0\right)$ at $1.501 \mathrm{MeV}$ and 1.369 is represented by the solid red line. As for magnesium isotopes ${ }^{30} \mathrm{Mg}$ is represented by the solid black line for transition $\left(J_{i}^{\pi} T_{i}=0^{+} 0\right)$ to the $\left(J_{f}^{\pi} T_{f}=4^{+} 0\right)$ at $3.901 \mathrm{MeV}$. The black dashed line represents the transition $\left(J_{i}^{\pi} T_{i}=0^{+} 0\right)$ to the $\left(J_{f}^{\pi} T_{f}=4^{+} 0\right)$ for ${ }^{32} \mathrm{Mg}$ at $2.246 \mathrm{MeV}$. The transition $\left(J_{i}^{\pi} T_{i}=0^{+} 0\right)$ to the $\left(J_{f}^{\pi} T_{f}=4^{+} 0\right)$ for ${ }^{34} \mathrm{Mg}$ at $2.193 \mathrm{MeV}$ is represented by blue solid line. Te curve shows the convergence of the experimental results of the isotope of magnesium ${ }^{24} \mathrm{Mg}$ and the theoretical results of the even isotopes of magnesium.

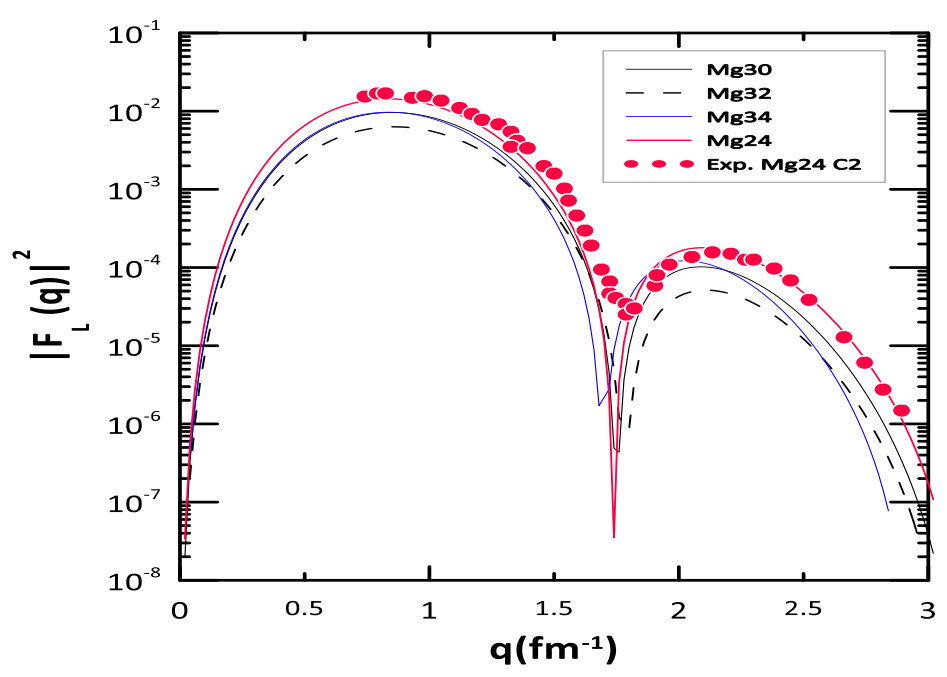

Fig. 9: The relation between moment transfer and form longitudinal factor for ${ }^{24,30,32,34} \mathrm{Mg}$ nuclei.

Fig. 10 shows the relation between moment transfer and transverses form factor. The theoretical results of the isotopes of magnesium ${ }^{25,29,31} \mathrm{Mg}$ were compared with Alzubadi, et al. [11] experimental results(the red circles) at $1.612 \mathrm{MeV}$ of the magnesium isotope ${ }^{25} \mathrm{Mg}$. The transition $\left(J_{i}^{\pi} T_{i}=5 / 2^{+} 1 / 2\right)$ to the $\left(J_{f}^{\pi} T_{f}=\right.$ $7 / 2^{+} 1 / 2$ ) for ${ }^{25} \mathrm{Mg}$ at $1.739 \mathrm{MeV}$ is represented by red solid line. The transition for 
${ }^{29} \mathrm{Mg}$ from $\left(J_{i}^{\pi} T_{i}=3 / 2^{+} 1 / 2\right)$ to the $\left(J_{f}^{\pi} T_{f}=1 / 2^{+} 1 / 2\right)$ at $0.088 \mathrm{MeV}$ is represented by black solid line. As for the magnesium isotope ${ }^{31} \mathrm{Mg}$ the transition $\left(J_{i}^{\pi} T_{i}=1 / 2^{+} 1 / 2\right)$ to the $\left(J_{f}^{\pi} T_{f}=3 / 2^{+} 1 / 2\right)$ at $0.176 \mathrm{MeV}$ is represented by black dashed line. When comparing the theoretical results with the experimental results for ${ }^{25} \mathrm{Mg}$ a mismatch was noticed. Thus other effects are needed to be added in order to obtain better results.

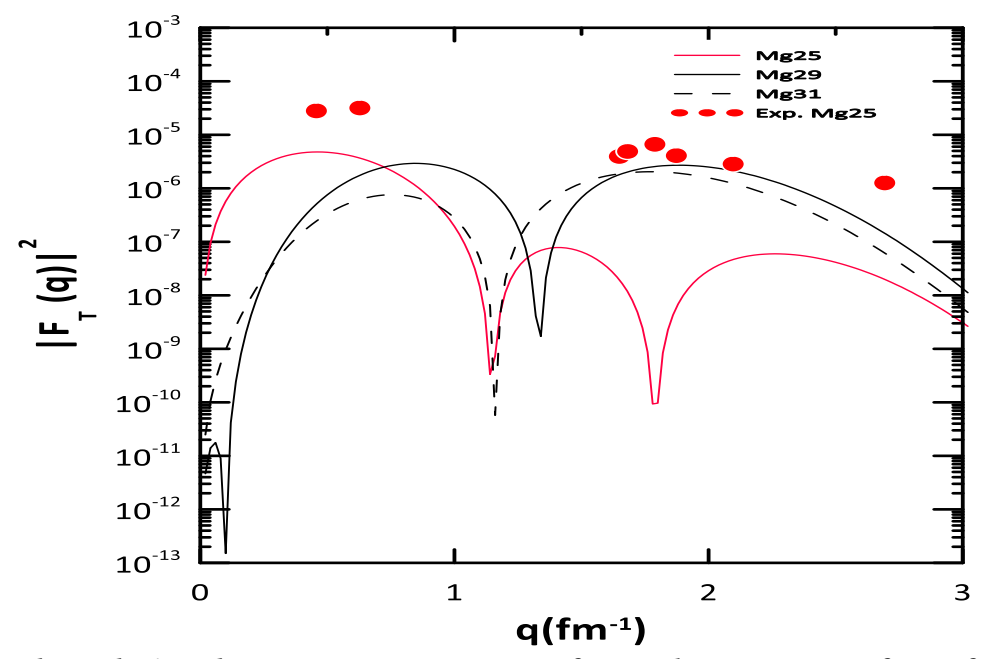

Fig. 10: The relation between moment transfer and transverses form factor for ${ }^{25,29,31} \mathrm{Mg}$ nuclei.

Fig. 11 shows the relation between moment transfer and transverses form factor. It shows comparison between the theoretical results of the isotopes of magnesium ${ }^{24,30,32,34} \mathrm{Mg}$. The transition for ${ }^{24} \mathrm{Mg}\left(J_{i}^{\pi} T_{i}=0^{+} 0\right)$ to the $\left(J_{f}^{\pi} T_{f}=2^{+} 0\right)$ at 1.501 $\mathrm{MeV}$ is represented by red solid line. For ${ }^{30} \mathrm{Mg}$ magnesium isotope, the transition $\left(J_{i}^{\pi} T_{i}=0^{+} 0\right)$ to the $\left(J_{f}^{\pi} T_{f}=2^{+} 0\right)$ at $3.901 \mathrm{MeV}$ is represented by black solid line. The transition $\left(J_{i}^{\pi} T_{i}=0^{+} 0\right)$ to the $\left(J_{f}^{\pi} T_{f}=2^{+} 0\right)$ for ${ }^{32} \mathrm{Mg}$ at $2.246 \mathrm{MeV}$ is represented by black dashed line. The transition $\left(J_{i}^{\pi} T_{i}=0^{+} 0\right)$ to the $\left(J_{f}^{\pi} T_{f}=2^{+} 0\right)$ for ${ }^{34} \mathrm{Mg}$ at $2.193 \mathrm{MeV}$ is represented by blue solid line.

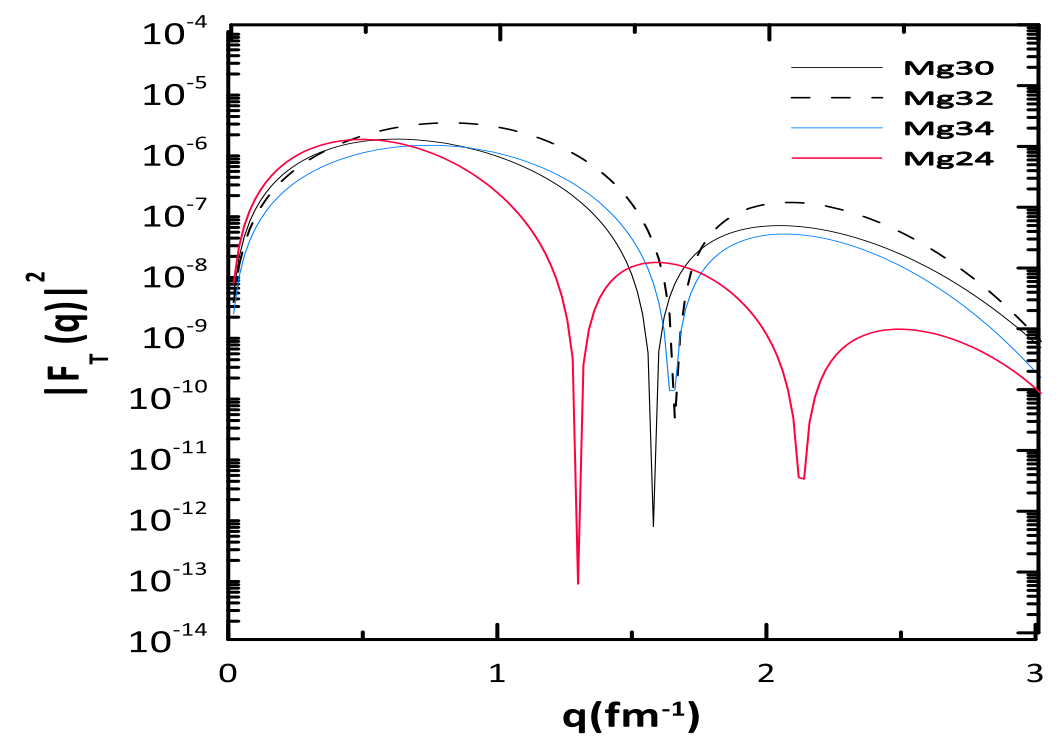

Fig. 11: The relation between moment transfer and transverses form factor for ${ }^{24,30,32,34} \mathrm{Mg}$ nuclei. 


\section{Conclusions}

In the present work, the microscopic structures of ${ }^{29-34} \mathrm{Mg}$-isotopes is investigated. Note the effect of the number of nucleons as the number of nucleons increased the root mean square for neutron charge, neutron skin thickness and radius for (protons, neutrons and matter) increased and when the number of nucleons decreased the (matter, proton, neutron and charge) density decreased. The convergence of experimental and theoretical results of even nuclei for longitudinal form factor was noted

\section{Acknowledgement}

The author's thank the head of the Department of Physics and thank the University of Baghdad, College of Science, Department of Physics for their help during the research work.

\section{References}

[1] PE Hodgson, E Gadioli, EG Erba "Introductory Nuclear Physics", Oxford University Press, 1997.

[2] John R. Lamarsh and Anthony J. Baratta, "Introduction to Nuclear Engineering", Third Edition, Prentice-Hall, Inc. Upper Saddle River, New Jersey, 2001.

[3] H. T. Fortune and R. Sherr, Physical Review, C86 (2012) 064322-1_064322-3.

[4] M. Bouhelal, N. Saidane, S. Belaid, F. Haas, Can. J. Phys., 96, 7 (2018).

[5] Rafah I. Noori and Arkan R. Ridha, Iraqi Journal of Science, 60, 6 (2019) 12861296.

[6] A. N. Antonov, P. E.Hodgson, I. Zh. Petkov, "Nucleon Momentum and Density Distribution in Nuclei", Clarendon Press, Oxford, (1988).

[7] T. De Forest Jr. and J. D. Walecka, Adv. Phys., 15, 57 (1966) 1-109.

[8] N.F. Mott., Proc. Roy. Soc. Ser., A124 (1929) 425-442.

[9] B. A. Brown, R. A. Radhi, B. H. Wildenthal; Phys. Rep., 101, 5 (1983) 313-358.

[10] G. C. Li, M. R. Yearian, I. Sick, Phys. Rev., C 9, 5 (1974) 1861-1877.

[11] Ali A. Alzubadi, Ali H. Taqi, Eman M. Rasheed, Iraqi Journal of Physics, 14, 31 (2016) 28-36. 